\title{
Die Tabubrecherin
}

JULIA VON DALL'ARMI Die Kunsttherapeutin Julia von Dall'Armi wählte für ihre Diplomarbeit das Thema „Die Weitergabe traumatisierender Erfahrungen am Beispiel des Zweiten Weltkrieges“. Kein leichter Stoff. Doch sie stellte sich ihm, weil seine Relevanz bis in die Gegenwart reicht.

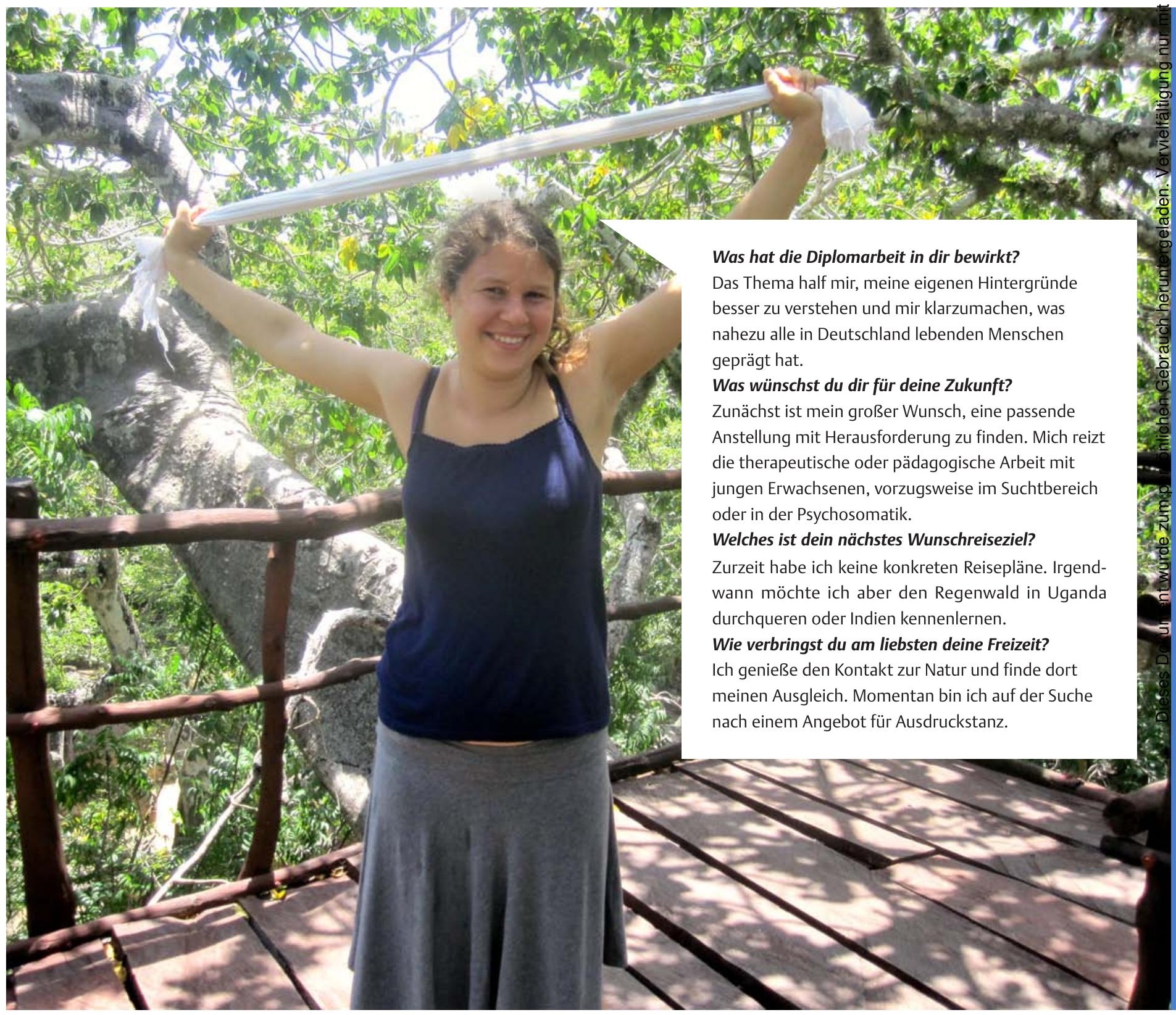




\section{Die Weitergabe traumatisierender Erfahrungen aus dem Zweiten Weltkrieg}

Julia von Dall'Armi ...

...geboren 1986 in der Nähe von Basel in der Schweiz, wuchs ab dem Alter von 10 Jahren in der Hansestadt Hamburg auf. Dort machte sie 2005 ihr Abitur. Danach zog es sie in die Ferne, nach Südafrika, wo sie im Rahmen eines sozialen Jahres in einer Einrichtung für Kinder mit Behinderung erstmals in Kontakt zu ihrem zukünftigen Beruf kam. Sie lernte die Kunsttherapie kennen und fand nach ihrer Rückkehr den passenden Studiengang an der Fachhochschule Ottersberg, der weltweit ältesten Hochschule für Kunsttherapie und -pädagogik. Während des Studiums legte sie ihren Schwerpunkt auf die Malerei, vorzugsweise mit Eitempera. Im theoretischen Teil fokussierte sie sich auf psychodynamische Zusammenhänge. Mit dem Diplomabschluss im Gepäck reiste sie 2011 erneut nach Afrika: vier Wochen Backpacking durch Kenia, größtenteils auf eigene Faust. Um sich für den Beruf zu rüsten, sammelt sie zurzeit als Jahrespraktikantin im Gemeinschaftskrankenhaus Havelhöhe wertvolle Erfahrungen in der kunsttherapeutischen Arbeit.

\section{Die Diplomarbeit}

Julia von Dall'Armi ist durch die Verdrängungen der Großeltern nicht mit deren Erfahrungen aus der Nazizeit in Kontakt gekommen. Weder in der Familie noch im Geschichtsunterricht bot sich ihr Raum für die Auseinandersetzung mit der eigenen Betroffenheit, nur die Fakten wurden gelehrt. In der therapeutischen Arbeit wurde ihr durch Erzählungen von Patienten klar, dass viele Zusammenhänge in den Tiefen der Vergangenheit versteckt liegen.

Um diese Zusammenhänge zu verstehen, ging Julia von Dall'Armi in ihrer Diplomarbeit der Frage nach, wie es sein kann, dass selbst noch junge Generationen in Bezug auf den Nationalsozialismus von Gefühlen wie Scham und Schuld betroffen sind. In ihrer Literaturrecherche konzentrierte sie sich auf psychologische Erklärungsmodelle. Dabei beleuchtete sie insbesondere die Täterrolle wie die von Massenmördern, Mitläufern oder passiven Zuschauern. Sie fand heraus, dass sich der dynamische Kreislauf zwischen Täter und Opfer, also das wechselseitige Spannungsverhältnis, das durch die Vermeidung der inneren Auseinandersetzung mit den Geschehnissen entsteht, in den nachfolgenden Generationen fortsetzt.

$\Rightarrow$ v. Dall'Armi Freiin v. Massenbach J. Die Weitergabe traumatisierender Erfahrungen am Beispiel des Zweiten Weltkrieges. Diplomarbeit an der Fachhochschule Ottersberg; 2011

\section{Ergebnisse}

Julia von Dall'Armi fand heraus, dass...

> die Täter in der Regel bis heute unter vernichtenden Gewissensschamgefühlen und nicht anerkannter realer Schuld leiden.

$>$ alle Mitglieder einer Täterfamilie durch „Loyalität" miteinander verbunden sind und die Nachkommen beeinflussen. Dadurch verfestigen sich destruktive Muster, was die persönliche Entwicklung der Kinder enorm einschränken kann. Das kann beispielsweise dazu führen, dass ein Kind, das sich stark mit dem traumatisierten Elternteil identifiziert, in einen Identitätskonflikt gerät.

$>$ ein widersprüchlicher Umgang der Großeltern mit der Nazizeit die Verarbeitung der Geschehnisse bei den nachfolgenden Generationen behindert. Sie verleugnen zum Beispiel, bagatellisieren, trauern um den Führer oder erwähnen positive Aspekte der NS-Zeit. Dem liegt eine Vermeidung der innerlichen Auseinandersetzung und somit des verantwortlichen Handelns zugrunde.

\section{Fazit}

Resümierend hält Julia von Dall'Armi fest, ... $>$ dass es für den Einzelnen unumgänglich ist, sich die schwerwiegende Vergangenheit mit ihrer Bedeutung und Konsequenz bewusst zu machen, um die Geschehnisse wirklich verarbeiten zu können.

$>$ dass es durch eine bewusste Auseinandersetzung mit den Tätern, Opfern und deren komplexer Verstrickungen gelingen kann, sich von den Belastungen der familiären Vergangenheit zu befreien und den Kreislauf zu durchbrechen. Das heißt, Befreiung ist möglich, wenn man sich den Geschehnissen der Vergangenheit stellt.

$>$ dass ein verantwortlicher Umgang das Annehmen der Geschichte bedeutet. Im Kleinen ist das eine bewusste Haltung zu sich selbst und zu seinen eigenen Erfahrungen. Besonders wichtig ist das in der Rolle als Therapeutin und in der Arbeit mit Patienten.

$>$ dass therapeutische Angebote auf dem Weg der Auf- und Verarbeitung unterstützen können: die Traumatherapie aus systemtheoretischer Sicht, die Familienaufstellung oder andere Methoden der systemischen Aufstellungsarbeit sowie körperorientierte Therapieansätze und die Psychotherapie. Die Kunsttherapie kann den kreativen Ausdruck seelischen Erlebens begleiten.

Nora Sieweke 